\title{
Randomized, Double-Blinded, Placebo-Controlled Trial of Fibrinogen Concentrate Supplementation After Complex Cardiac Surgery
}

Marco Ranucci, MD; Ekaterina Baryshnikova, PhD (Biol.); Giulia Beatrice Crapelli, MD; Niels Rahe-Meyer, MD; Lorenzo Menicanti, MD; Alessandro Frigiola, MD; for the Surgical Clinical Outcome REsearch (SCORE) Group*

Background-Postoperative bleeding after heart operations is still a common finding, leading to allogeneic blood products transfusion. Fibrinogen and coagulation factors deficiency are possible determinants of bleeding. The experimental hypothesis of this study is that a first-line fibrinogen supplementation avoids the need for fresh frozen plasma (FFP) and reduces the need for any kind of transfusions.

Methods and Results-This was a single-center, prospective, randomized, placebo-controlled, double-blinded study. One-hundred sixteen patients undergoing heart surgery with an expected cardiopulmonary bypass duration $>90$ minutes were admitted to the study. Patients in the treatment arm received fibrinogen concentrate after protamine administration; patients in the control arm received saline solution. In case of ongoing bleeding, patients in the treatment arm could receive prothrombin complex concentrates (PCCs) and those in the control arm saline solution. The primary endpoint was avoidance of any allogeneic blood product. Patients in the treatment arm had a significantly lower rate of any allogeneic blood products transfusion (odds ratio, 0.40 ; $95 \%$ confidence interval, 0.19 to $0.84, P=0.015)$. The total amount of packed red cells and FFP units transfused was significantly lower in the treatment arm. Postoperative bleeding was significantly $(P=0.042)$ less in the treatment arm (median, $300 \mathrm{~mL}$; interquartile range, 200 to $400 \mathrm{~mL}$ ) than in the control arm (median, $355 \mathrm{~mL}$; interquartile range, 250 to $600 \mathrm{~mL}$ ).

Conclusions-Fibrinogen concentrate limits postoperative bleeding after complex heart surgery, leading to a significant reduction in allogeneic blood products transfusions. No safety issues were raised.

Clinical Trial Registration-URL: http://www.clinicaltrials.gov. Unique identifier: NCT01471730. (J Am Heart Assoc. 2015;4: e002066 doi: 10.1161/JAHA.115.002066)

Key Words: cardiopulmonary bypass • fibrinogen • hemorrhage • surgery

I n complex cardiac surgery, operations with prolonged cardiopulmonary bypass (CPB), allogeneic blood products transfusions are often necessary to control perioperative bleeding and reverse excessive hemodilution. Fresh frozen plasma (FFP), platelet concentrates (PLT), and cryoprecipitate (CRYO) are the most commonly blood-derived procoagulant

From the Department of Cardiothoracic, Vascular Anesthesia and Intensive Care (M.R., E.B., G.B.C.) and Department of Cardiac Surgery (L.M., A.F.), IRCCS Policlinico San Donato, Milan, Italy; Clinic for Anesthesiology and Intensive Care Medicine, Hannover Medical School, Hannover, Germany (N.R.-M.).

${ }^{*} \mathrm{~A}$ list of members of the Surgical and Clinical Outcome Research (SCORE) Group has been provided in the Appendix.

Correspondence to: Marco Ranucci, MD, Department of Anesthesia and Intensive Care, IRCCS Policlinico San Donato, Via Morandi 30, 20097 San Donato Milanese, Milan, Italy. E-mail: cardioanestesia@virgilio.it

Received April 15, 2015; accepted May 5, 2015.

(C) 2015 The Authors. Published on behalf of the American Heart Association, Inc., by Wiley Blackwell. This is an open access article under the terms of the Creative Commons Attribution-NonCommercial License, which permits use, distribution and reproduction in any medium, provided the original work is properly cited and is not used for commercial purposes. agents, and packed red cells (PRCs) are used to maintain hemoglobin levels. FFP is the most commonly prescribed hemostatic agent, ${ }^{1}$ with more than 300000 units annually transfused in the $U^{2}$ and approximately 4.5 million units in the United States in $2011{ }^{3}$ without any sign of decrease over the last 10 years. $^{4}$

At present, FFP can be replaced by separate substitutes (albumin, antithrombin, coagulation factors, fibrinogen concentrate, and so on), depending on the different clinical and therapeutic needs. In the setting of bleeding control in complex cardiac surgery, the main reason for using FFP should be bleeding control in case of inadequate concentration/activity of coagulation factors and/or fibrinogen. A low activity of many coagulation factors is detectable after complex cardiac surgery, even if it may not be associated with postoperative bleeding. ${ }^{5}$ Conversely, low levels of fibrinogen after cardiac surgery have been associated with increased postoperative bleeding in different studies. ${ }^{5-7}$ Using FFP to correct low values of coagulation factors and/or fibrinogen may represent an inefficient treatment, given that it 
has been shown that large doses (up to 2 L) of FFP are required especially to correct fibrinogen deficits. ${ }^{8,9}$ Fibrinogen levels may be corrected using CRYO, but again large doses are required, and the availability of CRYO is limited or absent in many countries. ${ }^{10}$

The present study is aimed to verify the hypothesis that a FFP-free strategy, based on a first-line fibrinogen concentrate supplementation and a (facultative) second-line prothrombin complex concentrate (PCC) supplementation, is effective in reducing any kind of allogeneic blood product transfusions after complex heart surgery.

\section{Methods}

\section{Study Design and Population}

This is a prospective, randomized, double-blind, placebocontrolled, parallel-group, clinical study conducted at a single institution (IRCCS Policlinico San Donato, San Donato Milanese, Milan, Italy). The study was approved by the local ethics committee of Melegnano (Milan) ASL2 and by the Italian Agency of Drugs. The study was assigned the EudraCT code 2011-0046-33 and was registered at ClinicalTrials.gov with the identifier code NCT01471730.

The study was spontaneous, with the external support of CSL Behring, which provided the study drugs (fibrinogen concentrate and PCCs) and the ROTEM ${ }^{\circledR}$ reagents free of charge. CSL Behring had no role in data collection, analysis, and quality control. Data property belongs to the IRCCS Policlinico San Donato. All patients signed a written informed consent to participate in the study. The study began on November 29, 2011.

Eligible patients had to fulfill the following criteria: age 18 years or older; planned complex cardiac surgery operation with expected CPB duration of 90 minutes or longer; and presence of at least one of the following risk factors ${ }^{11}$ : age $>65$ years; non-elective surgery; serum creatinine level $>1.36 \mathrm{mg} / \mathrm{dL}$; and redo surgery.

Exclusion criteria were: participation in another randomized, controlled trial; refusal to participate; emergency surgery; known congenital or acquired coagulopathy; baseline antithrombin levels <80\%; known autoimmune disorder; pregnancy; and major antiplatelet agents $\left(P_{2} Y_{12}\right.$ inhibitors) not discontinuated in the 5 days before surgery. In patients with $P_{2} Y_{12}$ inhibitors discontinuated between 6 and 10 days before surgery, platelet function was tested and the patient was admitted to surgery (and to the study) according to predefined cut-off values as per our routine practice. ${ }^{12,13}$ Additional exclusion criteria were included to avoid the risk of transfusions triggered by preoperative anemia and/or excessive intraoperative hemodilution. These included a preoperative hematocrit (HCT) value $<35 \%$ and a body surface area $<1.7 \mathrm{~m}^{2}$.
During surgery, withdrawal criteria were established again to avoid hemodilution-related transfusions. Intraoperative PRC transfusions before study drug administration and excessive hemodilution during CPB ( $\mathrm{HCT}<23 \%)$ were withdrawal criteria. Patients screened and not withdrawn were randomized to the treatment arm or the control arm.

\section{Surgery and CPB}

All the patients received our standard surgical treatment and CPB technique. This included the use of reduced priming volume of the oxygenator and circuit, vacuum-assisted drainage, use of cell-saver intraoperatively, and use of postoperative systems to collect, process, and reinfuse chest drain blood. All patients received a total intraoperative dose of $30 \mathrm{mg} / \mathrm{kg}$ of tranexamic acid. CPB was established after a loading dose of $300 \mathrm{lU} / \mathrm{kg}$ of unfractionated heparin plus additional doses to reach and maintain a target activated clotting time of 450 seconds or longer.

\section{Interventions}

Patients were randomly allocated to a treatment arm or a placebo ( $0.9 \%$ saline solution) arm. Twenty minutes before the removal of the aortic cross-clamp, patients were tested with a ROTEM $^{\circledR}$ (TEM International, Munich, Germany) tissue-factor activated (EXTEM) and fibrin-based thromboelastometry test (FIBTEM). Clotting time (CT; seconds) at EXTEM and maximum clot firmness (MCF; mm) at the EXTEM and FIBTEM were recorded. After protamine administration (1:1 ratio heparin loading dose-protamine dose), patients in the treatment arm received fibrinogen concentrate (Haemocomplettan ${ }^{\circledR}$; CSL Behring, Marburg, Germany) at a dose that depended on the MCF at the FIBTEM, according to the previously published equation $^{14,15}$ :

$$
\begin{aligned}
& \text { Fibrinogen concentrate dose }(\mathrm{g}) \\
& \begin{aligned}
= & (\text { target FIBTEM MCF }[22 \mathrm{~mm}] \\
& - \text { actual FIBTEM MCF }[\mathrm{mm}]) \\
& \times(\text { bodyweight }[\mathrm{kg}] / 140)
\end{aligned}
\end{aligned}
$$

After 15 minutes from fibrinogen concentrate (or placebo) administration and in the presence of ongoing microvascular bleeding, a second EXTEM was performed, and in case of a CT longer than 80 seconds, the patients of the study arm were planned to receive 4-factors PCCs (Confidex ${ }^{\circledR}$; CSL Behring, Marburg, Germany) at a dose of $7 \mathrm{U} / \mathrm{kg}$, whereas patients in the control arm received placebo treatment.

In the presence of ongoing microvascular bleeding intra- or postoperatively, bleeding and anemia control was guaranteed by our standard protocol recently published in a modified version, ${ }^{16}$ which considers: 
1. FFP if the international normalized ratio (INR) $>1.5$ or the reaction time at thromboelastography (Haemoscope, Niles, IL) in a heparinase cup (to rule out residual heparin effects) $>12$ minutes.

2. Platelet concentrate in the case of a platelet count $<50000$ cells $/ \mu \mathrm{L}$.

3. PRCs (1 unit at a time) under the following conditions: always if the hemoglobin $(\mathrm{Hb})$ value $<7 \mathrm{~g} / \mathrm{dL}$; possible without medical justification if $\mathrm{Hb}$ value between 7 and $7.9 \mathrm{~g} / \mathrm{dL}$; possible but with medical justification (hemodynamic instability; oxygen extraction rate $>40 \%$; signs of organ ischemia) if $\mathrm{Hb}$ value between 8 and $9 \mathrm{~g} / \mathrm{dL}$; never if $\mathrm{Hb}$ value $>9 \mathrm{~g} / \mathrm{dL}$.

In the presence of bleeding refractory to the above treatment, determining severe hemodynamic instability, and for all the life-saving conditions, an empirical rescue therapy was allowed, inclusive of the use PCCs, fibrinogen concentrate, and recombinant activated factor VII. Surgical revision was considered in the presence of ongoing bleeding without signs of coagulopathy at the viscoelastic and conventional laboratory tests.

\section{Measurements}

Data collection was performed using our institutional database, which includes all the demographics, preoperative factors, procedure details, intraoperative data, and outcome measurements. Transfusion risk was assessed using a transfusion risk score, ${ }^{17}$ whereas the overall severity of the patients' profile was assessed using the logistic EuroSCORE II. ${ }^{18}$ For the purpose of the present study, the following additional data have been collected: FIBTEM MCF; EXTEM CT; fibrinogen values at the arrival in the intensive care unit (ICU; (Clauss method); and platelet count at the arrival in the ICU.

\section{Study Endpoints}

The primary endpoint of this study was avoidance of any allogeneic blood products usage (inclusive of PRCs, FFP, and PLT). CRYO were not considered, given that it is not available at our institution. For the primary endpoint, the window of observation was hospital stay up to 30 days.

Secondary endpoints were: number of PRC, FFP, and PLT units (same window of observation as for the primary endpoint); massive blood transfusion (7 units or more of PRCs in the first 24 postoperative hours); postoperative bleeding (chest drain output in the first 12 postoperative hours); and surgical revision as a result of bleeding (first 48 postoperative hours).

Safety endpoints were operative mortality and thromboembolic complications (in-hospital or within 30 days from surgery after hospital discharge).

\section{Sample Size}

Sample size was based on the primary endpoint (allogeneic blood products transfusions). Historical data from the last 5 years were retrieved from the institutional database by selection of the patients fulfilling the inclusion/exclusion/ withdrawal criteria of the present study. Allogeneic blood products transfusion rate was $61 \%$. Effect size was settled at a transfusion rate of $35 \%$ for the treatment arm and $60 \%$ for the control arm. With a 2-sided alpha value of 0.05 and beta value of 0.20 , the sample size was calculated at 116 patients (58 in each arm). Considering a withdrawal rate of $3 \%$, the number of patients to be enrolled was settled at 120 .

\section{Randomization and Masking}

A randomization code was generated with a computerized system. Sealed envelopes containing the randomization codes were prepared. The randomization ratio of the treatment arms was 1:1. The randomization process was managed by an unblinded biologist (E.B.).

The unblinded biologist was in charge of drug or placebo preparation, which was performed in a laboratory annexed to the operating theater. The FIBTEM/EXTEM tests were done in the same laboratory by the same biologist. Drug or placebo were prepared in sterile bags after performing the FIBTEM/ EXTEM tests. Each gram of fibrinogen concentrate was diluted in $50 \mathrm{~mL}$ of sterile water or an equivalent volume of $0.9 \%$ saline solution as placebo. The same procedure was followed for PCCs. Medication(s) were delivered to the operating room after protamine administration (fibrinogen concentrate or placebo) or at the request of the attending anesthesiologist in bleeding patients (PCCs or placebo). The attending anesthesiologist, the surgical staff, and doctors in charge for the clinical care in the ICU and ward were blinded, as well as the person in charge of the institutional database data entry.

\section{Statistics}

All data are expressed as number and percentage; continuous variables are expressed as mean with SD for normally distributed variables and as median with interquartile range (IQR) for non-normally distributed variables.

Efficacy and safety analysis of between-arms differences were based on a Pearson chi-square for frequency differences, on a Student $t$ test for differences in continuous, normally distributed variables, and on nonparametric tests (MannWhitney $U$ test) for differences in continuous, non-normally distributed variables.

Post-hoc analyses included linear or nonlinear regression models, aimed to determine the association between 
postoperative bleeding and coagulation tests and between treatment, transfusion risk, CPB time, and transfusions. Additional descriptive analyses on severely bleeding patients were performed.

An intention-to-treat analysis was applied. All the statistical analyses were performed with a computerized package (SPSS 13.0; IBM, Chicago, IL).

\section{Results}

\section{Patients and Procedures}

The general screening, enrollment, and randomization procedure is shown in Figure 1. The first patient was randomized on November 30, 2011 and the last on December 10, 2014. Three patients were withdrawn after enrollment: 1 patient did not receive surgery, and the other 2 were transfused before completion of CPB. All patients received the allocated treatment (fibrinogen or placebo); no patient in the treatment arm fulfilled the criteria for the receiving PCCs, and 4 patients in the placebo arm underwent the second treatment and received placebo.

Adherence to the transfusion protocol was $100 \%$ in both arms. The final analysis for efficacy and safety include 58 patients in each arm.
Baseline clinical and demographic characteristics of patients are shown in Table 1. There was no significant imbalance between groups for any variable.

Based on the values of the FIBTEM MCF measured 20 minutes before aortic cross-clamp removal and the equation above reported, patients in the treatment arm received fibrinogen concentrate at a median dose of $4 \mathrm{~g}$ (IQR, 3 to 6).

\section{Coagulation-Related Laboratory Data}

Table 2 reports coagulation-related laboratory data, as measured during the procedure (ROTEM) and at arrival in the ICU. After dosing, there was a significant $(P=0.001)$ shortening of clotting time at the EXTEM in the treatment arm and no changes in the control arm. This led to a significant between-groups difference. Patients in the treatment arm demonstrated a significant increase in clot firmness both at EXTEM and FIBTEM, whereas patients in the control arm did not. At the arrival in the ICU, patients in the treatment arm had significantly higher levels of fibrinogen, as measured by the central laboratory.

\section{Efficacy Endpoints}

Primary and secondary efficacy endpoints are reported in Table 3. During hospital stay, 39 (67.2\%) patients in the

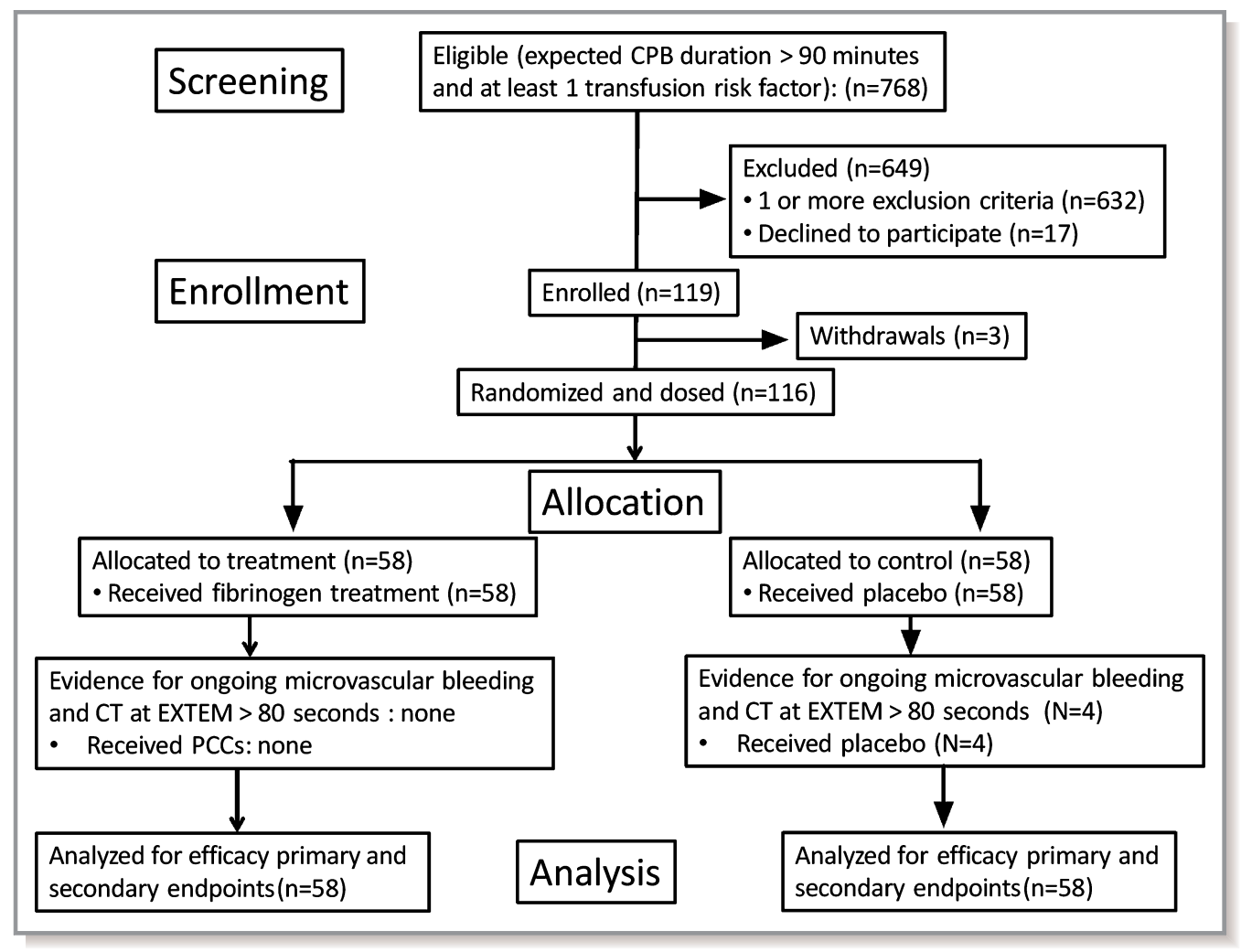

Figure 1. Diagram showing the flow of participants through each stage of the trial. CPB indicates cardiopulmonary bypass; CT, clotting time; EXTEM, tissue-factor activated thromboelastometry test; PCCs, prothrombin complex concentrates. 
Table 1. Baseline Clinical and Demographic Characteristics

\begin{tabular}{|c|c|c|c|}
\hline & Intervention Arm & Control Arm & $P$ Value \\
\hline Age, y & 72 (64 to 76 ) & 73 (68 to 79$)$ & 0.128 \\
\hline Weight, kg & 74.5 (13.4) & 74.7 (15.3) & 0.939 \\
\hline $\begin{array}{l}\text { Body surface } \\
\text { area, } \mathrm{m}^{2}\end{array}$ & $1.85(0.19)$ & $1.86(0.20)$ & 0.808 \\
\hline Gender male & $41(71)$ & $43(74)$ & 0.678 \\
\hline $\begin{array}{l}\text { Left ventricular } \\
\text { ejection fraction, \% }\end{array}$ & 57 (50 to 60$)$ & 57 (45 to 63 ) & 0.600 \\
\hline $\begin{array}{l}\text { Congestive heart } \\
\text { failure }\end{array}$ & $6(10)$ & $10(17)$ & 0.281 \\
\hline Active endocarditis & $1(2)$ & $1(2)$ & 1.000 \\
\hline $\begin{array}{l}\text { Serum creatinine, } \\
\mathrm{mg} / \mathrm{dL}\end{array}$ & 1.0 (0.8 to 1.1$)$ & 1.05 (0.8 to 1.2 ) & 0.199 \\
\hline $\begin{array}{l}\text { Serum bilirubin, } \\
\mathrm{mg} / \mathrm{dL}\end{array}$ & 0.5 (0.5 to 0.8$)$ & 0.5 (0.5 to 0.7$)$ & 0.810 \\
\hline Hematocrit, \% & $40.3(3.2)$ & $40.0(2.9)$ & 0.577 \\
\hline $\begin{array}{l}\text { Chronic obstructive } \\
\text { pulmonary disease }\end{array}$ & $4(7)$ & $7(12)$ & 0.342 \\
\hline $\begin{array}{l}\text { Previous } \\
\text { cerebrovascular } \\
\text { accident }\end{array}$ & $2(3)$ & $4(7)$ & 0.402 \\
\hline $\begin{array}{l}\text { Diabetes on } \\
\text { medication }\end{array}$ & $4(7)$ & $10(17)$ & 0.087 \\
\hline Redo surgery & $12(21)$ & $8(14)$ & 0.326 \\
\hline Non-elective surgery & $2(3)$ & $4(7)$ & 0.402 \\
\hline $\begin{array}{l}\text { Transfusion risk } \\
\text { score }\end{array}$ & 15 (10 to 17$)$ & 15 (13 to 17$)$ & 0.278 \\
\hline EuroSCORE ॥ & 3.7 (2 to 5.6 ) & 4.7 (2 to 8.4$)$ & 0.196 \\
\hline \multicolumn{4}{|l|}{ Type of surgery } \\
\hline $\mathrm{CABG}+$ mitral valve & $8(14)$ & $9(15)$ & 0.793 \\
\hline $\mathrm{CABG}+$ aortic valve & $13(22)$ & $13(22)$ & 1.000 \\
\hline $\begin{array}{l}\mathrm{CABG}+\mathrm{LV} \\
\text { aneurysmectomy }\end{array}$ & $4(7)$ & $5(9)$ & 0.729 \\
\hline Double/triple valve & $9(15)$ & $8(14)$ & 0.793 \\
\hline Ascending aorta & $12(21)$ & $16(28)$ & 0.385 \\
\hline Others & $12(21)$ & $7(12)$ & 0.210 \\
\hline $\begin{array}{l}\text { Cardiopulmonary } \\
\text { bypass } \\
\text { time, min }\end{array}$ & $105(39)$ & $111(40)$ & 0.367 \\
\hline $\begin{array}{l}\text { Lowest hematocrit } \\
\text { on } \mathrm{CPB}\end{array}$ & $28.5(3.3)$ & $27.8(3.5)$ & 0.294 \\
\hline $\begin{array}{l}\text { Total heparin dose, } \\
I U \times 100\end{array}$ & 245 (210 to 300$)$ & 240 (200 to 271$)$ & 0.424 \\
\hline $\begin{array}{l}\text { Total protamine } \\
\text { dose, } \\
\text { mg }\end{array}$ & 250 (200 to 300 ) & 245 (200 to 280 ) & 0.973 \\
\hline
\end{tabular}

Data are number (percentage) or median (interquartile range) or mean (SD). CABG indicates coronary artery bypass graft; CPB, cardiopulmonary bypass; IU, international units; LV, left ventricle. treatment arm were free from any allogeneic blood product transfusions, a significantly $(P=0.012)$ higher rate than those in the control arm (44.8\%). The absolute difference in transfusion avoidance was $13(22.4 \%)$ patients (relative difference for transfusion avoidance, 1.6; 95\% confidence interval [Cl], 1.07 to 2.42 ).

Among the secondary endpoints, postoperative bleeding, as recorded from chest drains during the first 12 postoperative hours, was significantly lower in the treatment arm, and the total amount of PRCs, FFP, and PLT used during hospital stay was significantly lower. When limited to patients receiving PRC transfusions, the difference in transfused units between the 2 arms was not statistically different.

Figure 2 shows the total amount of allogeneic blood products transfused in the 2 study arms from the end of CPB through the whole postoperative hospital stay.

\section{Safety}

Overall, the patient population experienced a morbidity/ mortality rate that is compatible with the complexity of the surgical procedure. The overall mortality rate was $3.6 \%$, lower than the predicted mortality rate according to the EuroSCORE II (5.6\%; $95 \% \mathrm{Cl}, 4.5$ to 6.7$)$, with $1(1.8 \%)$ event in the treatment arm and $3(5.3 \%)$ in the control arm. The patient who died in the treatment arm had a postoperative low cardiac output refractory to drug therapy and intra-aortic balloon pump, developed a systemic blood infection with multiorgan failure, received a tracheostomy, and finally died after 34 days postsurgery.

Postoperative morbidity was observed in 34 (60.7\%) of the patients in the treatment arm and $36(64.3 \%)$ in the control arm. Adverse events observed were typical for patients undergoing this kind of surgery, without between-groups differences. Thromboembolic complications were specifically investigated and are presented in Table 4. There were no differences between groups; none of the adverse events observed was considered related to the study drug(s). No acute reaction to study drug(s) administration was observed.

\section{Post-Hoc Analyses}

Patients with severe postoperative bleeding were identified according to the Universal Definition of Perioperative Bleeding ${ }^{19}$ as those who had a postoperative chest drain loss $>1000 \mathrm{~mL} / 12 \mathrm{~h}$. These were 5 patients: 4 in the control arm and 1 in the treatment arm. Preoperative standard coagulation values were in the normal range in all these patients, with an aPTT between 31.3 and 35 seconds, an INR between 1.07 and 1.16, and PLT count between 150000 and 212000 cells/ $\mu \mathrm{L}$. The preoperative HCT ranged from $35 \%$ to $39.3 \%$. No patient was under warfarin or oral anticoagulants. Two 
Table 2. Coagulation Data During the Procedure and at the Arrival in the ICU

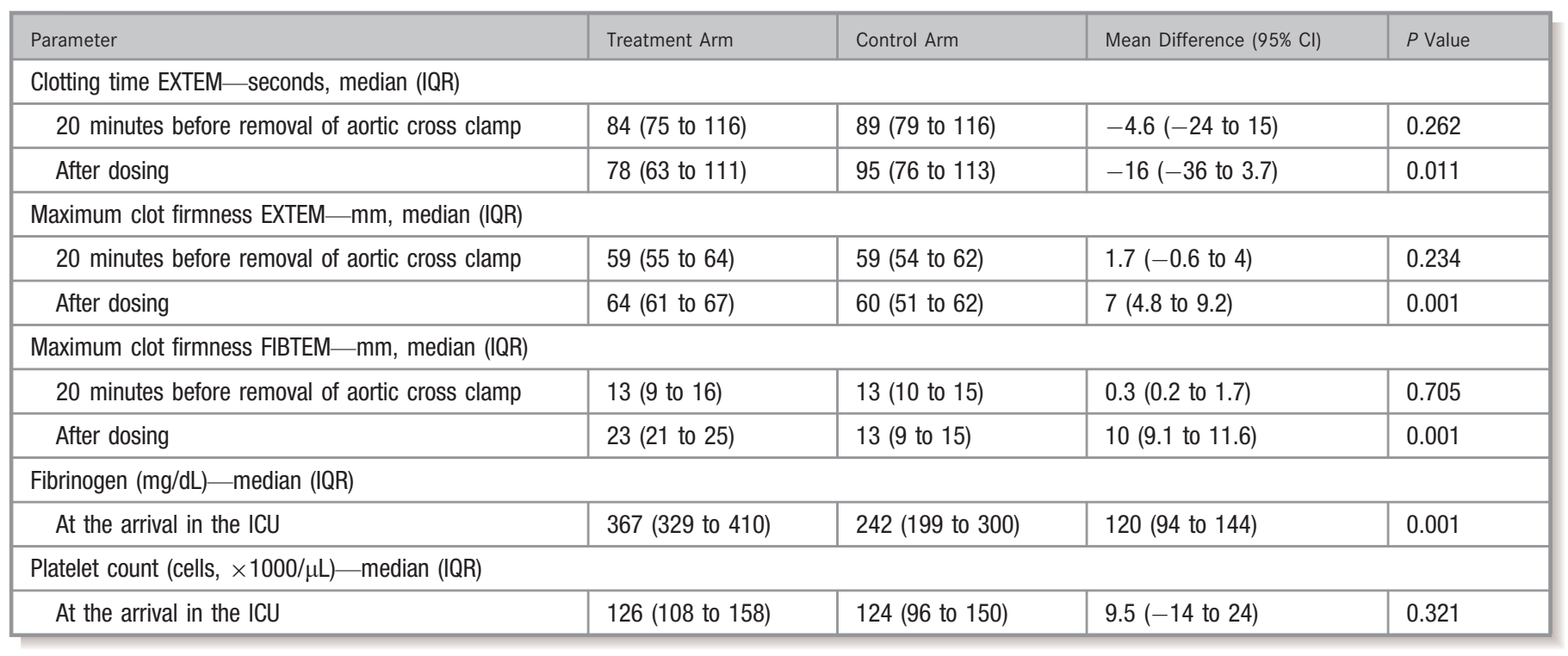

$\mathrm{CI}$ indicates confidence interval; EXTEM, tissue-factor activated thromboelastometry test; FIBTEM, fibrin-based thromboelastometry test; ICU, intensive care unit; IQR, interquartile range.

patients were redo cases; 3 patients received ascending aorta surgery, 1 coronary artery bypass graft (CABG)+mitral valve repair, and 1 mitral valve and aortic valve replacement. Only 1 patient required surgical revision. Two patients in the control arm were treated with PCCs on top of FFP and PLT concentrates.

After correction for transfusion risk score and CPB duration, the treatment arm remained independently associ- ated with transfusion avoidance in a multivariable logistic regression analysis, with an odds ratio (OR) of $0.43(95 \% \mathrm{Cl}$, 0.19 to $0.98, P=0.046)$. For a fixed CPB duration of 90 minutes, the absolute difference in transfusion risk at 4 different levels of transfusion risk score $(5,10,15$, and 20 points) was $6 \%, 12 \%, 19 \%$, and $21 \%$, respectively.

A sensitivity analysis aimed to determine the association between postdosing viscoelastic tests/fibrinogen values and

Table 3. Efficacy Endpoints

\begin{tabular}{|c|c|c|c|c|}
\hline Endpoint & Treatment Arm & Control Arm & OR (95\% Cl) & $P$ Value \\
\hline \multicolumn{5}{|l|}{ Avoidance of allogeneic blood products } \\
\hline Packed red cells — no. of patients (\%) & $39(67.2)$ & $26(44.8)$ & $0.40(0.19$ to 0.84$)$ & 0.015 \\
\hline Fresh frozen plasma — no. of patients (\%) & $58(100)$ & $50(86.2)$ & $\mathrm{N} / \mathrm{A}$ & 0.006 \\
\hline \multicolumn{5}{|l|}{ Secondary endpoints } \\
\hline \multicolumn{5}{|l|}{ Transfusions (overall patient population) } \\
\hline Packed red cells — no. of units, median (IQR) & 0 (0 to 1$)$ & $1(0$ to 2$)$ & $\mathrm{N} / \mathrm{A}$ & 0.010 \\
\hline Fresh frozen plasma — no. of units, median (IQR) & 0 (0 to 0$)$ & $0(0$ to 0$)$ & $\mathrm{N} / \mathrm{A}$ & 0.002 \\
\hline Postoperative bleeding — mL/12 h, median (IQR) & $300(200$ to 400$)$ & 355 (250 to 600$)$ & $\mathrm{N} / \mathrm{A}$ & 0.042 \\
\hline Massive red blood cells transfusion & $0(0)$ & $2(3.4)$ & $\mathrm{N} / \mathrm{A}$ & 0.496 \\
\hline Surgical revision & $0(0)$ & $2(3.4)$ & $\mathrm{N} / \mathrm{A}$ & 0.496 \\
\hline
\end{tabular}

$\mathrm{Cl}$ indicates confidence interval; IQR, interquartile range; N/A, not applicable; OR, odds ratio. 


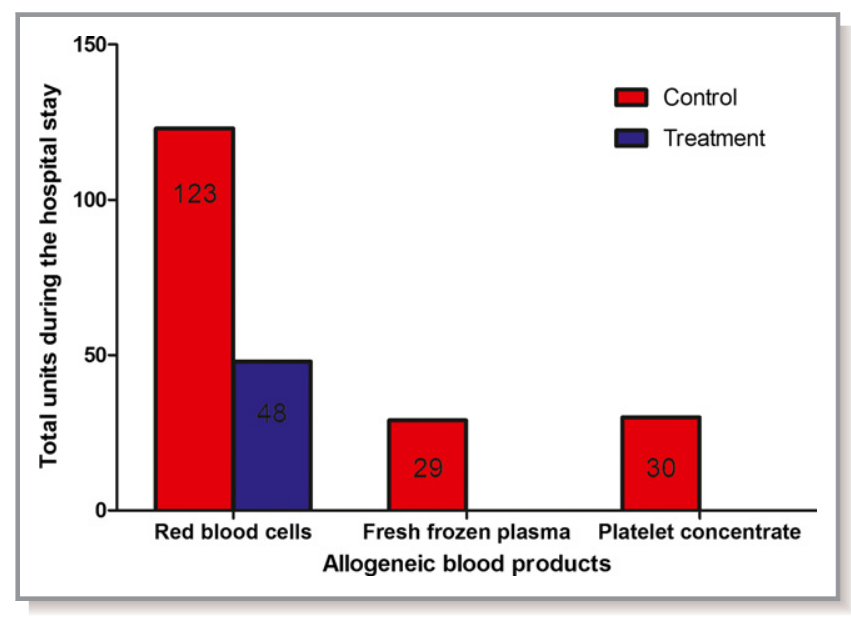

Figure 2. Total amount of allogeneic blood products transfused in the 2 study arms from the end of cardiopulmonary bypass to hospital discharge.

chest drain output in the first 12 postoperative hours was applied. Linear and nonlinear regression analyses were performed, and the best fit was found for a power equation (Figure 3). There was a significant $(P=0.001)$ negative association between fibrinogen values at arrival in the ICU and postoperative bleeding. Similarly, there was a significant $(P=0.008)$ negative association between MCF at FIBTEM and postoperative bleeding. The negative relationship between

Table 4. Safety Endpoints

\begin{tabular}{|l|l|l|l|}
\hline Event & Treatment Arm & Control Arm & $P$ Value \\
\hline Any kind of complication & $35(60.3)$ & $38(65.5)$ & 0.564 \\
\hline Low cardiac output & $9(15.5)$ & $14(24.1)$ & 0.244 \\
\hline Atrial fibrillation & $24(41.4)$ & $24(41.4)$ & 1.000 \\
\hline Lung dysfunction & $1(1.7)$ & $0(0)$ & 0.315 \\
\hline Acute kidney injury & $5(8.6)$ & $6(10.3)$ & 0.751 \\
\hline Infections & $3(5.2)$ & $4(6.9)$ & 0.697 \\
\hline Bloodstream & $0(0)$ & $0(0)$ & 1.000 \\
\hline Pneumonia & $4(6.9)$ & $7(12.1)$ & 0.342 \\
\hline Urinary tract & $0(0)$ & $0(0)$ & 1.000 \\
\hline Wound & $0(0)$ & $0(0)$ & 1.000 \\
\hline Mediastinitis & \multicolumn{3}{|l}{} \\
\hline Thromboembolic events & $0(0)$ & $0(0)$ & 1.000 \\
\hline Myocardial infarction & $0(0)$ & $0(0)$ & 1.000 \\
\hline Stroke & $0(0)$ & $0(0)$ & 1.000 \\
\hline Peripheral thromboembolism & $0(0)$ & $0(0)$ & 1.000 \\
\hline Pulmonary thromboembolism & $0(0)$ & $1(1.7)$ & 0.315 \\
\hline Mesenteric infarction & $1(1.7)$ & $3(5.2)$ & 0.309 \\
\hline Operative mortality & \multicolumn{5}{|l}{} \\
\hline
\end{tabular}

Data are number (\%).

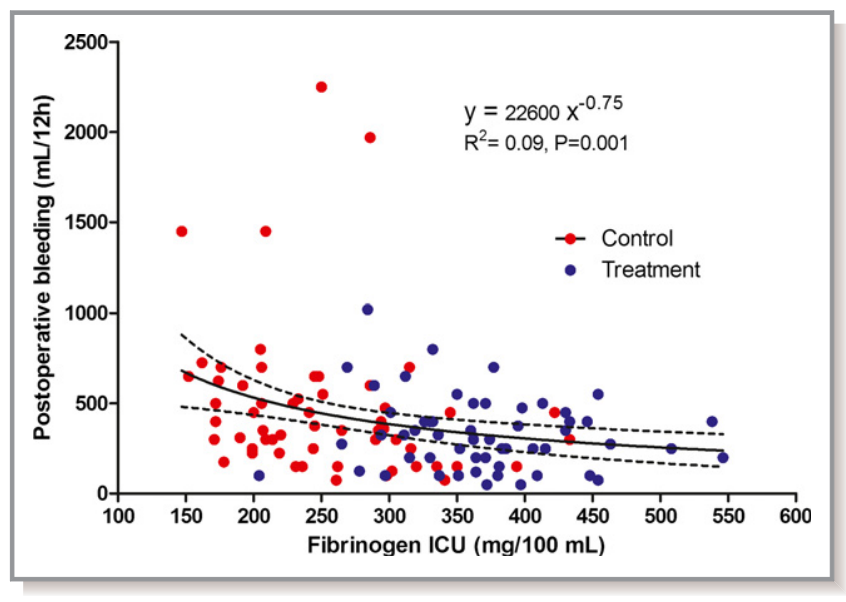

Figure 3. Fibrinogen levels and postoperative bleeding. Dashed lines are $95 \%$ confidence interval. ICU indicates intensive care unit.

fibrinogen levels at arrival in the ICU and postoperative bleeding remained significant $(P=0.05)$ after adjustment for the treatment arm.

No association was found between CT at EXTEM and postoperative bleeding.

\section{Discussion}

The results of the present study can be summarized as follows: (1) In the setting of complex cardiac surgery with CPB, FFP transfusions could be completely avoided using a strategy based on purified concentrates of coagulation factors; (2) within this strategy, fibrinogen alone was needed to control postoperative bleeding, without any patient requiring PCCs; and (3) this approach resulted in a significantly lower need for PRC transfusions, lower amount of allogeneic blood products transfused, and lower postoperative bleeding.

Our study confirms the findings of previous small, randomized, controlled trials and prospective studies where an early use of fibrinogen concentrate was associated with a bleeding containment and less allogeneic blood products (especially FFP) use. $^{20-22}$ Of notice, no patient in the treatment arm required PCCs. Our zero-plasma protocol was based on a first-line supplementation with fibrinogen concentrate, and only as a second step the patients would have received PCCs, in case of ongoing bleeding and prolonged clotting time at EXTEM. This condition was never observed in treatment arm patients and rarely in control arm patients. A deficiency of coagulation factors (excluding fibrinogen and factor XIII) leads to a decreased thrombin generation with prolonged CT at EXTEM, whereas a deficiency in fibrinogen leads to decreased clot firmness with reduced MCF at EXTEM and FIBTEM. Previous studies have highlighted that this second mechanisms is associated with postoperative 
bleeding in cardiac surgery, whereas the first is not. ${ }^{5}$ Our data support this interpretation, with 3 additional data emerging from the viscoelastic and conventional tests before and after fibrinogen administration: (1) Fibrinogen replenishment actually not only increases clot firmness, but also even reduces CT; (2) no association exists between CT and postoperative bleeding; and (3) there is a significant association between fibrinogen levels at arrival in the ICU and postoperative bleeding.

Of notice, patients in the control arm rarely experienced very low postoperative fibrinogen levels (only 1 patient $<150 \mathrm{mg} / 100 \mathrm{~mL}$ and 11 patients $<200 \mathrm{mg} / 100 \mathrm{~mL}$ ). Despite this, raising fibrinogen levels with fibrinogen concentrate was effective in reducing chest drain blood loss. This result was obtained without reaching abnormally high fibrinogen levels (only 3 patients between 500 and $550 \mathrm{mg} /$ $100 \mathrm{~mL}$ ).

Our results were confirmative of an association between postoperative fibrinogen levels and bleeding tendency. However, the relationship was not linear, and within a wide range of postoperative fibrinogen values (between 280 and $550 \mathrm{mg} / \mathrm{dL}$ ), there was no association; below the value of $280 \mathrm{mg} / \mathrm{dL}$, the relationship acquired significance and all the severely bleeding patients belonged to this region.

According to a post-hoc analysis, the efficacy of prophylactic fibrinogen supplementation was more evident in patients at higher bleeding risk, with an absolute reduction in transfusion rate that reached $21 \%$ when the transfusion risk score exceeded 20 points. The present study is a "proof of concept" that replacing FFP with fibrinogen concentrate is an effective strategy for avoiding allogeneic blood product transfusion in complex heart surgery; however, it is not intended to suggest that all patients undergoing complex heart surgery should be prophylactically treated with fibrinogen at the end of CPB. The combination of complex surgery with a high transfusion risk score is probably the best environment to consider this option.

Postoperative bleeding and transfusion needs are both multifactorial and complex items in cardiac surgery. Postoperative bleeding may result from a number of factors, including low PLT count or PLT function defects, deficiency of fibrinogen and soluble coagulation factors, and residual effects of heparin, hyperfibrinolysis, and surgical sources. ${ }^{23}$ Studies dealing with prohemostatic agents having postoperative bleeding/transfusions as the primary endpoint must deal with this multifactorial mechanism underlying bleeding during and after cardiac surgery and should minimize the effects of confounders.

In our protocol, we have applied a number of procedures to minimize the effects of potential confounders, as well as to better stress the effects of the study variable (first-line use of fibrinogen concentrate and only second-line use of PCCs to replace FFP). Patients were operated on over a 3-year period by the same 4 surgeons, thus minimizing surgeon-related variability in bleeding control. Additionally, a patient blood management program has been established in our institution for many years, based on preoperative assessment of PLT function, intraoperative minimization of hemodilution, postoperative use of point-of-care coagulation tests (thromboelastography), and the application of a strict transfusion protocol. Overall, the patients in this study have been treated according to our usual policy of patient blood management, therefore following the philosophy of a pragmatic randomized, controlled trial. Within our strategy, a number of measures minimized the effects of possible confounders. Decision making on the timing of surgery in patients under double antiPLT therapy was based on PLT function tests, rather than on an arbitrary discontinuation time. ${ }^{12,13}$ All patients received tranexamic acid to control hyperfibrinolysis. Presence of residual heparin effects after protamine administration was ruled out with specific tests with and without heparinase.

Transfusion of allogeneic blood products is strongly institution dependent, as demonstrated by the high intercenter variability in a recent randomized, controlled trial, where only the hospital where surgery took place was a predictor of transfusion rate. ${ }^{24}$ This particularly applies to red blood cell transfusions, which may be triggered by anemia and hemodilution rather than by bleeding. To this respect, we tried to limit the potential confounding effect of these factors, excluding or withdrawing patients at high risk for receiving PRC transfusion as a result of non-bleeding-related anemia. ${ }^{24}$ Moreover, the standard application of a well-established transfusion protocol (without protocol violations) strongly reduced the attending physician-related variability in the allogeneic blood product transfusions.

Overall, our reduction of the "background noise" that is quite typical in transfusion and bleeding-based studies probably created the adequate environment for assessing the efficacy of the zero-plasma strategy, leading to the achievement of the primary endpoint.

Among the secondary endpoints, we believe that the evidence of a postoperative bleeding containment associated with the zero-plasma strategy is of particular relevance. The potentially beneficial effects of replacing FFP with fibrinogen concentrate rely on a better control of bleeding tendency, and no direct effect of this strategy in limiting allogeneic blood products transfusions could be advocated in the absence of a significant decrease in blood loss.

From the safety point of view, no concerns were raised with respect to possible adverse effects related to fibrinogen supplementation, with no significant between-groups differences in morbidity events. The administration of prohemostatic drugs in the setting of cardiac surgery is always burdened by the potential risk of thromboembolic 
complications, as reported with the use of activated ${ }^{25}$ and nonactivated ${ }^{26}$ coagulation factors. Our study confirms previous reports about the safety of fibrinogen concentrate supplementation. Our series of treated patients reached values of MCF at the EXTEM that never exceeded the upper limit of the reference range,$^{27}$ with an increased contribution of fibrinogen compensating for the reduced post-CPB PLT count and function. However, no patient in our study actually received both fibrinogen concentrate and PCCs. Therefore, we cannot exclude that this combination of factor concentrates may actually result in a greater risk of thromboembolic complications.

Our study has a number of limitations. The first is its single-center protocol, which may be seen both as a strength (by reducing experimental variability) or a weakness (the zero-plasma strategy may be seen as effective only if applied in a specific center with a number of additional strategies already in place). A second limitation is the exclusion of patients with anemia and small body surface area; albeit justified by the need to avoid confounding factors determining non-bleeding-related transfusions, this patient selection limits the generalizability of our findings. A third point pertains the timing of fibrinogen supplementation. Our protocol is based on a first-line, pre-emptive strategy of fibrinogen replenishment in patients at high risk for postoperative bleeding, before bleeding takes place. It is not the intention of the present study to suggest routine fibrinogen administration in this subgroup of patients: We decided to follow a prophylactic strategy to investigate the efficacy of a zero-plasma approach in a controlled environment and not to wait until excessive bleeding was diagnosed. Studies based on a postoperative bleeding trigger for testing the efficacy of prohemostatic drugs are carried by a screening failure rate $>80 \%{ }^{25}$ that is unacceptable in a single-center trial.

It is our feeling and clinical practice not to start treating postoperative bleeding before it appears clinically relevant (even if this may delay the onset of treatment leading to less beneficial effects), nor to treat numbers rather than bleeding. The suggestion from our study is that in the presence of excessive bleeding after heparin neutralization, associated with low fibrinogen levels, fibrinogen supplementation may be an adequate choice, avoiding the need for PCCs and/or FFP. An exact cut-off point for fibrinogen levels triggering fibrinogen supplementation is still to be determined, and its finding is outside the study purposes. Larger patient populations are required to determine at which level of functional (FIBTEM) fibrinogen or conventional (Clauss) fibrinogen value fibrinogen supplementation is required.

Another point of debate is the adequate fibrinogen concentrate dose to achieve a clinically relevant effect. Our protocol is based on data from previous studies ${ }^{14,15}$ where a target MCF value of $22 \mathrm{~mm}$ at FIBTEM was proposed. This led to a median dose of $4 \mathrm{~g}$ of fibrinogen concentrate supplementation, which is more than the dose that we usually consider (2 g), but, however, much less than the dose reported in other studies ${ }^{19}(8 \mathrm{~g})$. The dose used in our study and the target value of $22 \mathrm{~mm}$ for the MCF at FIBTEM have been effective in reducing bleeding and postoperative transfusion needs, avoiding the use of PCCs and FFP. However, it is possible that lower targets of MCF, as well as lower doses of fibrinogen concentrate, may be effective as well.

In conclusion, our study demonstrates the efficacy of a zero-plasma strategy in complex cardiac surgery, based on a first-line treatment with fibrinogen concentrate. No safety issues related to this strategy were raised. Open questions remain with respect to the cost benefit of this approach, trigger values of fibrinogen concentration, and fibrinogen concentrate dose to be used.

\section{Appendix}

The Surgical and Clinical Outcome Research (SCORE) group includes the following members: Marco Ranucci, MD, FESC, San Donato Milanese (Milan); Alessandro Frigiola, MD, San Donato Milanese (Milan); Lorenzo Menicanti, MD, San Donato Milanese (Milan); Serenella Castelvecchio, MD, FESC, San Donato Milanese (Milan); Ekaterina Baryshnikova, PhD (Biol), San Donato Milanese (Milan); Valeria Pistuddi, San Donato Milanese (Milan); Santi Trimarchi, MD, San Donato Milanese (Milan); Concetta Carlucci, MD, San Donato Milanese (Milan); Alessandro Varrica, MD, San Donato Milanese (Milan); Angela Satriano, MD, San Donato Milanese (Milan); Maria Teresa La Rovere, MD, FESC, Montescano (Pavia).

\section{Acknowledgments}

Author Contributions: Ranucci, corresponding author, confirms that he had full access to all the data in the study and had final responsibility for the decision to submit for publication. Ranucci designed the study, analyzed the data, and wrote the manuscript; Baryshnikova participated in the study as the unblinded biologist; Crapelli participated in data collection and analysis; Rahe-Meyer contributed to study design and manuscript revision; and Menicanti and Frigiola contributed to study design, data interpretation, and manuscript preparation.

\section{Sources of Funding}

This study was funded by local research funds of the IRCCS Policlinico San Donato. CSL Behring provided the study drugs and the ROTEM reagents free of charge. 


\section{Disclosures}

Dr Ranucci reports personal fees from CSL Behring and personal fees from TEM International, outside the submitted work. Dr Rahe-Meyer reports grants and personal fees from CSL Behring (Marburg, Germany) and grants and personal fees from MSD (Whitehouse Station, NJ) outside the submitted work. No conflicts of interest for the other authors.

\section{References}

1. Puetz J. Fresh frozen plasma: the most commonly prescribed hemostatic agent. J Thromb Haemost. 2013;11:1794-1799.

2. Stanworth SJ, Grant-Casey J, Lowe D, Laffan M, New H, Murphy MF, Allard S. The use of fresh-frozen plasma in England: high levels of inappropriate use in adults and children. Transfusion. 2011;51:62-70.

3. US Department of Health and Human Services. The 2009 National Blood Collection and Utilization Survey Report. Washington, DC: US Department of Health and Human Services, Office of the Assistant Secretary for Health; 2011. Available at: http://www.hhs.gov/ash/bloodsafety/2011-nbcus.pdf. Accessed December 16, 2014.

4. Vonk AB, Meesters MI, van Dijk WB, Eijsman L, Romijn JW, Jansen EK, Loer SA, Boer C. Ten-year patterns in blood product utilization during cardiothoracic surgery with cardiopulmonary bypass in a tertiary hospital. Transfusion. 2014;54:2608-2616.

5. Ternstrom L, Radulovic V, Karlsson M, Baghaei F, Hyllner M, Bylock A, Hansson $\mathrm{KM}$, Jeppsson A. Plasma activity of individual coagulation factors, hemodilution and blood loss after cardiac surgery: a prospective observational study. Thromb Res. 2010;126:e128-e133.

6. Kindo M, Hoang Minh T, Gerelli S, Perrier S, Meyer N, Schaeffer M, Bentz J, Announe T, Mommerot A, Collange O, Pottecher J, Cristinar M, Thiranos JC, Gros H, Mertes PM, Billaud P, Mazzucotelli JP. Plasma fibrinogen level on admission to the intensive care unit is a powerful predictor of postoperative bleeding after cardiac surgery with cardiopulmonary bypass. Thromb Res. 2014;134:360-368.

7. Reinhöfer M, Brauer M, Franke U, Barz D, Marx G, Lösche W. The value of rotation thromboelastometry to monitor disturbed perioperative haemostasis and bleeding risk in patients with cardiopulmonary bypass. Blood Coagul Fibrinolysis. 2008;19:212-219.

8. Yang L, Stanworth S, Baglin T. Cryoprecipitate: an outmoded treatment? Transfus Med. 2012;22:315-320.

9. Collins PW, Solomon C, Sutor K, Crispin D, Hochleitner G, Rizoli S, Schöchl H, Schreiber M, Ranucci M. Theoretical modelling of fibrinogen supplementation with therapeutic plasma, cryoprecipitate, or fibrinogen concentrate. $\mathrm{Br} J$ Anaesth. 2014;113:585-595.

10. Ranucci M, Solomon C. Supplementation of fibrinogen in acquired bleeding disorders: experience, evidence, guidelines, and licences. $\mathrm{Br} /$ Anaesth. 2012;109:135-137.

11. Alghamdi AA, Davis A, Brister S, Corey P, Logan A. Development and validation of Transfusion Risk Understanding Scoring Tool (TRUST) to stratify cardiac surgery patients according to their blood transfusion needs. Transfusion. 2006;46:1120-1129.

12. Ranucci M, Baryshnikova E, Soro G, Ballotta A, De Benedetti D, Conti D; Surgical and Clinical Outcome Research (SCORE) Group. Multiple electrode whole-blood aggregometry and bleeding in cardiac surgery patients receiving thienopyridines. Ann Thorac Surg. 2011;91:123-129.

13. Ranucci M, Colella D, Baryshnikova E, Di Dedda U; Surgical and Clinical Outcome Research (SCORE) Group. Effect of preoperative P2Y12 and thrombin platelet receptor inhibition on bleeding after cardiac surgery. $\mathrm{Br} J$ Anaesth. 2014;113: 970-976.

14. Rahe-Meyer N, Pichlmaier M, Haverich A, Solomon C, Winterhalter M, Piepenbrock S, Tanaka KA. Bleeding management with fibrinogen concentrate targeting a high-normal plasma fibrinogen level: a pilot study. $\mathrm{Br} J$ Anaesth. 2009; 102:785-792.

15. Rahe-Meyer N, Solomon C, Winterhalter M, Piepenbrock S, Tanaka K, Haverich A, Pichlmaier M. Thromboelastometry-guided administration of fibrinogen concentrate for the treatment of excessive intraoperative bleeding in thoracoabdominal aortic aneurysm surgery. J Thorac Cardiovasc Surg. 2009; 138:694-702.

16. Song HK, von Heymann C, Jespersen CM, Karkouti K, Korte W, Levy JH, Ranucci M, Saugstrup T, Sellke FW. Safe application of a restrictive transfusion protocol in moderate-risk patients undergoing cardiac operations. Ann Thorac Surg. 2014;97:1630-1635.

17. Ranucci M, Castelvecchio S, Frigiola A, Scolletta S, Giomarelli P, Biagioli B. Predicting transfusions in cardiac surgery: the easier, the better: the Transfusion Risk and Clinical Knowledge score. Vox Sang. 2009;96:324-332.

18. Nashef SA, Roques F, Sharples LD, Nilsson J, Smith C, Goldstone AR, Lockowandt U. EuroSCORE II. Eur J Cardiothorac Surg. 2012;4:734-744.

19. Dyke C, Aronson S, Dietrich W, Hofmann A, Karkouti K, Levi M, Murphy GJ, Sellke FW, Shore-Lesserson L, von Heymann C, Ranucci M. Universal definition of perioperative bleeding in adult cardiac surgery. J Thorac Cardiovasc Surg. 2014;147:1458-1463.

20. Rahe-Meyer N, Solomon C, Hanke A, Schmidt DS, Knoerzer D, Hochleitner G, Sørensen B, Hagl C, Pichlmaier M. Effects of fibrinogen concentrate as firstline therapy during major aortic replacement surgery. Anesthesiology. 2013;118:40-50.

21. Görlinger K, Dirkmann D, Hanke AA, Kamler M, Kottenberg E, Thielmann M, Jakob H, Peters J. First-line therapy with coagulation factor concentrates combined with point-of-care coagulation testing is associated with decreased allogeneic blood transfusion in cardiovascular surgery: a retrospective, singlecenter cohort study. Anesthesiology. 2011;115:1179-1191.

22. Solomon C, Schöchl H, Hanke A, Calatzis A, Hagl C, Tanaka K, Rahe-Meyer N. Haemostatic therapy in coronary artery bypass graft patients with decreased platelet function: comparison of fibrinogen concentrate with allogeneic blood products. Scand J Clin Lab Invest. 2012;72:121-128.

23. Ranucci M. Hemostatic and thrombotic issues in cardiac surgery. Semin Thromb Hemost. 2015;41:84-90.

24. Karkouti K, von Heymann C, Jespersen CM, Korte W, Levy JH, Ranucci M, Sellke FW, Song HK. Efficacy and safety of recombinant factor XIII on reducing blood transfusions in cardiac surgery: a randomized, placebo-controlled, multicenter clinical trial. J Thorac Cardiovasc Surg. 2013;146:927-939.

25. Gill R, Herbertson M, Vuylsteke A, Olsen PS, von Heymann C, Mythen M, Sellke F, Booth F, Schmidt TA. Safety and efficacy of recombinant activated factor VII: a randomized placebo-controlled trial in the setting of bleeding after cardiac surgery. Circulation. 2009;120:21-27.

26. White R, Rushbrook J, McGoldrick J. The dangers of prothrombin complex concentrate administration after heart surgery. Blood Coagul Fibrinolysis. 2008; 19:609-610.

27. Lang T, Bauters A, Braun SL, Pötzsch B, von Pape KW, Kolde HJ, Lakner M. Multi-centre investigation on reference ranges for ROTEM thromboelastometry. Blood Coagul Fibrinolysis. 2005;16:301-310. 